\title{
Ranking of Districts of West Bengal by an Index Developed Through Probabilistic Approach Based on Household Data for Rural Areas of Census 2011
}

\author{
Partho Pratim Bhadra ${ }^{1}$, Tushar Kanti Ghara ${ }^{2}$ \\ ${ }^{1}$ Deputy Director, Directorate of Census Operations, West Bengal, Govt. of India \\ IB-199, Sector -III, Salt Lake, Kolkata 700106 \\ ${ }^{2}$ Joint Director of Public Instruction, Department of Higher Education, Govt. of West Bengal \\ $8^{\text {th }}$ Floor, Bikash Bhavan, Salt Lake City, Kolkata 700091
}

Corresponding Author: Tushar Kanti Ghara

\begin{abstract}
Population Census in India is conducted once in every ten years under the census Act 1948 and Census rules 1990 . The last decennial census was conducted in the year 2011. The three broad categories of Houselisting \& Housing Census are Housing condition, Amenities and Assets. Here, an attempt has been made to use four different approach using Probability theory for finding Ranks based on these House listing and Housing Census Data for rural areas. Although, there may be different Index developed to find such ranking, ranks obtained here using these different approaches are then compared and statistically tested. Statistical techniques like Probability, Weighted Geometric mean and Weighted Arithmetic mean, Correlation coefficient,and other Descriptive Statistical theories have been applied on this.
\end{abstract}

Keywords: Probability, Weighted Geometric mean, Proportionate weight, ranking, correlation

\section{INTRODUCTION}

India is a welfare State. Since independence, various welfare schemes have been launched for the welfare of the common man. This information is provided by the Census. Census in India is conducted in two phases, (i) House listing \& Housing Census is the $1^{\text {st }}$ Phase. and (ii) Population Census is the $2^{\text {nd }}$ phase. The Houselisting and Housing Census has immense utility as it provides comprehensive data on the conditions of human settlements, housing deficit and consequently the housing requirement to be taken care of in the formulation of housing policies. This also provide a wide range of data on amenities and assets available to the households, information much needed by various departments of the Union and State Governments and other non-Governmental agencies for development and planning at the local level as well as the State level. This also provide the base for Population Enumeration.For Census purposes, total geographical area is broadly classified into Rural \& Urban. The basic Unit of rural area is revenue Village (CENSUS OF INDIA, 2011). The rural data set is considered here for the State of West Bengal. The ranking of the districts have been calculated based on the individual values of each observations (Bhadra \& Ghara 2020). Different approach has been adopted earlier to this to rank the district based on the dataset.Household quality of living (HQL) refers to three broad aspects including housing condition, amenities and assets. The study is an attempt to investigate regional variation of the districts of West Bengal in terms of HQL based on published 2011 Census data (Das and Mistri 2013). There are about 69\% of the population lived in rural areas. States are compared using different methods based on household data (Panda 2014).

Here, an attempt is made to rank the districts of the state of West Bengal based on Rural data considering the Housing infrastructure, Availability of Household Amenities and Assets. Using the probability approach and then adopting the Simple Arithmetic mean, Simple Geometric mean, Weighted Arithmetic Mean and Weighted Geometric Mean the analytical process have been done, which is further tested by Correlation and Rank Test. The data analysis has been done by usingSPSS.

\section{DATA}

Census of India 2011 is used for the purpose of the study. The entire exercise is done on the Rural data set of House listing \& Housing Census which was conducted in 2010. The main variables are Housing infrastructure, Availability of Household Amenities and Assets. We consider here 11 such main variables and 78 sub variables. These 78 variables are actually sub variables of 11 main 


\section{Ranking of Districts of West Bengal by an Index Developed Through Probabilistic Approach Based on Household Data for Rural Areas of Census 2011}

variables. The data considered for rural West Bengal covering all districts except Kolkata. The breakup of those selected 11 main and $78 \mathrm{sub}$ variables are as below. The variables are - Census house (residence, residence-cum-other use, shop/office, school/ college/etc, hotel/ lodge/ guest house/etc, hospital/ dispensary/etc, factory/ workshop/ work shed/etc., place of worship, other nonresidential use);Condition of census houses (good, livable, dilapidated); Material of roof ( grass/thatch/bamboo/wood/mud/etc, plastic/polythene, handmade tiles, machine made tiles, burnt brick, stone/slate, GI/metal/ asbestos sheets, concrete, any other material); Material of wall (grass/ thatch/ bamboo/ wood/ mud/etc, plastic/ polythene, Mud/ Unburnt brick, Wood, Stone not packed with mortar, Stone packed with mortar, GI/metal/asbestos sheets, Burnt brick,concrete, any other material); Material of floor (mud, wood/bamboo, burnt brick, stone, cement, mosaic/floor tiles, any other material); Availability of assets (radio/transistor, television, computer/laptop , bicycle, scooter/motorcycle/moped, none of the assets,);Here the variable Computer/laptop reflects the total of Computer/laptop with Internet and Without Internet. In Census database, there is another asset termed as availability of Car/ Jeep/Van. This is also precluded in the analysis because of the uses of this asset varies significantly as someone is using it as luxury goods and someone is for his daily earnings and for this reason it won't be very justified to use this variable in this study. Main source of lighting (electricity, kerosene, solar energy, other oil, any other, no lighting);Type of latrine facility within the premises(piped sewer system, septic tank, other system of flush latrine, with slab/ventilated improved pit, without slab/open pit of Pit Latrine, night soil disposed into open drain, night soil serviced by animal)No latrine within premises (public latrine, open latrine);Number of households having bathing facility within the premises(bathroom, enclosure without roof) and no bathroom; Waste water outlet connected to (closed drainage, open drainage, no drainage); Cooking facility (has kitchen-fire-wood, crop residue, cow dung cake, coal/lignite/charcoal, kerosene, lpg/png, does not have kitchen -fire-wood, crop residue, cow dung cake, coal/lignite/charcoal, kerosene, lpg/png, and no cooking).

The sub variables under each main variable are weighted according to their importance in social structure.

Table 1.1: showing the weightage scheme(shown in the last column in each table)

\begin{tabular}{|c|c|c|c|c|c|c|c|c|}
\hline \multicolumn{9}{|c|}{ Uses of census houses(X1..) } \\
\hline Residence & $\begin{array}{l}\text { Residence } \\
\text {-cum- } \\
\text { other use }\end{array}$ & $\begin{array}{l}\text { Shop/ } \\
\text { Office. }\end{array}$ & $\begin{array}{l}\text { School/ } \\
\text { College } \\
\text { etc. }\end{array}$ & $\begin{array}{l}\text { Hotel/ } \\
\text { Lodge/ } \\
\text { Guest } \\
\text { house etc }\end{array}$ & $\begin{array}{l}\text { Hospital/ } \\
\text { Dispensary } \\
\text { etc.. }\end{array}$ & $\begin{array}{l}\text { Factory/ } \\
\text { Workshop/ } \\
\text { Workshed } \\
\text { etc. }\end{array}$ & $\begin{array}{l}\text { Place } \\
\text { of } \\
\text { worship. }\end{array}$ & $\begin{array}{l}\text { Other } \\
\text { non- } \\
\text { residential } \\
\text { use }\end{array}$ \\
\hline 4 & 3 & 5 & 6 & 7 & 8 & 9 & 2 & 1 \\
\hline
\end{tabular}

*Number of census houses used as Schools, Colleges, etc were more than 15 lakhs (From Census 2001)

\begin{tabular}{|l|l|l|}
\hline \multicolumn{3}{|l|}{ Condition if Census Houses X2.. } \\
\hline Good & Livable & Dilapidated \\
\hline 3 & 2 & 1 \\
\hline
\end{tabular}

*10 million households in the houses which were in dilapidated condition. (From Census 2001)

$* 50 \%$ of all the households in India categorized the condition of census houses occupied by them as 'Good'(From Census 2001)

* 44.3\% categorized as 'Livable'.( (From Census 2001))

\begin{tabular}{|l|l|l|l|l|l|l|l|l|}
\hline \multicolumn{2}{|l|}{ Material of Roof X3.. } \\
\hline $\begin{array}{l}\text { Grass/ Thatch/ } \\
\text { Bamboo/ } \\
\begin{array}{l}\text { Wood/ Mud, } \\
\text { etc.. }\end{array}\end{array}$ & $\begin{array}{l}\text { Plastic/ } \\
\text { Polythene. }\end{array}$ & $\begin{array}{l}\text { Handmade } \\
\text { Tiles. }\end{array}$ & $\begin{array}{l}\text { Machine } \\
\text { made } \\
\text { Tiles. }\end{array}$ & $\begin{array}{l}\text { Burnt } \\
\text { Brick. }\end{array}$ & Stone/Slate. & $\begin{array}{l}\text { G.I./ } \\
\text { Metal/ } \\
\text { Asbestos } \\
\text { sheets. }\end{array}$ & Concrete. & $\begin{array}{l}\text { Any } \\
\text { other } \\
\text { material. }\end{array}$ \\
\hline 2 & 3 & 4 & 6 & 7 & 8 & 5 & 9 & 1 \\
\hline
\end{tabular}

42 million or 22 percent household had roof made of grass, thatch, mud etc. (From Census 2001)

\begin{tabular}{|c|c|c|c|c|c|c|c|c|c|}
\hline \multicolumn{10}{|c|}{ Material of Wall X4.. } \\
\hline $\begin{array}{l}\text { Grass/ } \\
\text { Thatch/ } \\
\text { Bamboo } \\
\text { etc.. }\end{array}$ & $\begin{array}{l}\text { Plastic/ } \\
\text { Polythene. }\end{array}$ & $\begin{array}{l}\text { Mud/ } \\
\text { Unburnt } \\
\text { brick. }\end{array}$ & Wood. & $\begin{array}{l}\text { Stone } \\
\text { not } \\
\text { packed } \\
\text { with } \\
\text { mortar. }\end{array}$ & $\begin{array}{l}\text { Stone } \\
\text { packed } \\
\text { with } \\
\text { mortar. }\end{array}$ & $\begin{array}{l}\text { G.I./Metal/ } \\
\text { Asbestos } \\
\text { sheets. }\end{array}$ & $\begin{array}{l}\text { Burnt } \\
\text { brick. }\end{array}$ & Concrete. & $\begin{array}{l}\text { Any } \\
\text { other } \\
\text { material. }\end{array}$ \\
\hline 2 & 3 & 4 & 5 & 8 & 9 & 6 & 7 & 10 & 1 \\
\hline
\end{tabular}


Ranking of Districts of West Bengal by an Index Developed Through Probabilistic Approach Based on Household Data for Rural Areas of Census 2011

*61 million households had wall made of mud and unburnt bricks. (From Census 2001)

\begin{tabular}{|l|l|l|l|l|l|l|}
\hline \multicolumn{2}{|l|}{ Material of Floor } \\
\hline Mud & $\begin{array}{l}\text { Wood/ } \\
\text { Bamboo }\end{array}$ & Burnt Brick & Stone & Cement & $\begin{array}{l}\text { Mosaic/ } \\
\text { Floor } \\
\text { tiles }\end{array}$ & $\begin{array}{l}\text { Any } \\
\text { other } \\
\text { material }\end{array}$ \\
\hline 2 & 3 & 4 & 6 & 5 & 7 & 1 \\
\hline
\end{tabular}

*110 million or 57 percent households had Mud floors in the country (From Census 2001)

\begin{tabular}{|l|l|l|l|l|l|}
\hline \multicolumn{5}{|l|}{ Availability of assets } \\
\hline $\begin{array}{l}\text { Radio/ } \\
\text { Transist } \\
\text { or }\end{array}$ & $\begin{array}{l}\text { Televisi } \\
\text { on }\end{array}$ & $\begin{array}{l}\text { Computer/Lapt } \\
\text { op }\end{array}$ & Bicycle & $\begin{array}{l}\text { Scooter/ } \\
\text { Motorcycle/M } \\
\text { oped }\end{array}$ & $\begin{array}{l}\text { None of the assets } \\
\text { specified in col. } \\
10 \text { to 19 }\end{array}$ \\
\hline 2 & 4 & 6 & 3 & 5 & 1 \\
\hline
\end{tabular}

*6.62 crore out of 19.17 crore households in India did not possess any of the assets. In the beginning of this millennium 67 million [35.1\%]households had radio/ transistor. In the beginning of this millennium 61 million households had television (From Census 2001)

\begin{tabular}{|l|l|l|l|l|l|}
\hline \multicolumn{6}{|l|}{ Main Source of lighting } \\
\hline Electricity & Kerosene & $\begin{array}{l}\text { Solar } \\
\text { energy }\end{array}$ & Other oil & $\begin{array}{l}\text { Any } \\
\text { other }\end{array}$ & $\begin{array}{l}\text { No } \\
\text { lighting }\end{array}$ \\
\hline 6 & 4 & 5 & 3 & 2 & 1 \\
\hline
\end{tabular}

*In the rural the mainsourceswere kerosene (55.6\%) and electricity (43.5\%) (From Census2001)

\begin{tabular}{|c|c|c|c|c|c|c|c|c|}
\hline \multicolumn{7}{|c|}{ Type of latrine facility within the premises } & \multirow{2}{*}{\multicolumn{2}{|c|}{$\begin{array}{l}\begin{array}{l}\text { No latrine within } \\
\text { premises }\end{array} \\
\text { Alternative source }\end{array}$}} \\
\hline \multicolumn{3}{|c|}{$\begin{array}{lll}\begin{array}{l}\text { Flush/pour } \\
\text { connected to }\end{array} & & \text { flush } \\
\end{array}$} & \multicolumn{2}{|l|}{ Pit latrine } & \multirow{2}{*}{$\begin{array}{l}\text { Night soil } \\
\text { disposed } \\
\text { into open } \\
\text { drain }\end{array}$} & \multirow{2}{*}{$\begin{array}{l}\text { Service Latrine } \\
\text { Night soil serviced } \\
\text { by animal }\end{array}$} & & \\
\hline $\begin{array}{l}\text { Piped } \\
\text { sewer } \\
\text { system }\end{array}$ & $\begin{array}{l}\text { Septic } \\
\text { tank }\end{array}$ & $\begin{array}{l}\text { Other } \\
\text { system }\end{array}$ & $\begin{array}{l}\text { With slab/ } \\
\text { ventilated } \\
\text { improved pit }\end{array}$ & $\begin{array}{l}\text { Without } \\
\text { slab/ } \\
\text { open pit }\end{array}$ & & & $\begin{array}{l}\text { Public } \\
\text { latrine }\end{array}$ & Open \\
\hline 10 & 9 & 8 & 7 & 6 & 4 & 3 & 5 & 1 \\
\hline
\end{tabular}

*63.6\% households did not have any latrine within the house (From Census2001).

\begin{tabular}{|l|l|l|}
\hline \multicolumn{2}{|l|}{ Number of households having bathing facility within the premises } \\
\hline Yes & $\begin{array}{l}\text { Enclosure without } \\
\text { roof }\end{array}$ & No \\
\hline Bathroom & 2 & 1 \\
\hline 3 & &
\end{tabular}

*Only 6.93 crore out of 19.17 crore households in India had bathroom facility.(From Census2001)

\begin{tabular}{|l|l|l|}
\hline \multicolumn{2}{|l|}{ Waste water outlet connected to } \\
\hline Closed drainage & Open drainage & No drainage \\
\hline 3 & 2 & 1 \\
\hline
\end{tabular}

*More than half the households in the country did not have any drainage connectivity for waste water outlet.(From Census2001)

\begin{tabular}{|c|c|c|c|c|c|c|c|c|c|c|c|c|}
\hline \multicolumn{6}{|c|}{ Has Kitchen } & \multicolumn{6}{|c|}{ Does not have kitchen } & \multirow[b]{2}{*}{ No kitchen } \\
\hline $\begin{array}{l}\text { Fire- } \\
\text { wood }\end{array}$ & $\begin{array}{l}\text { Crop } \\
\text { residue }\end{array}$ & $\begin{array}{l}\text { Cow } \\
\text { dung } \\
\text { cake }\end{array}$ & $\begin{array}{l}\text { Coal, } \\
\text { Lignite, } \\
\text { Charcoal }\end{array}$ & $\begin{array}{l}\text { Ker } \\
\text { ose } \\
\text { ne }\end{array}$ & $\begin{array}{l}\text { LP } \\
\text { G/P } \\
\text { NG }\end{array}$ & $\begin{array}{l}\text { Fire- } \\
\text { wood }\end{array}$ & $\begin{array}{l}\text { Crop } \\
\text { residue }\end{array}$ & $\begin{array}{l}\text { Cow } \\
\text { dung } \\
\text { cake }\end{array}$ & $\begin{array}{l}\text { Coal, } \\
\text { Lignite, } \\
\text { Charcoal }\end{array}$ & $\begin{array}{l}\text { Ker } \\
\text { ose } \\
\text { ne }\end{array}$ & $\begin{array}{l}\mathrm{LPG} \\
/ \mathrm{PN} \\
\mathrm{G}\end{array}$ & \\
\hline 5 & 6 & 7 & 9 & 11 & 13 & 2 & 3 & 4 & 8 & 10 & 12 & 1 \\
\hline
\end{tabular}




\section{Ranking of Districts of West Bengal by an Index Developed Through Probabilistic Approach Based on Household}

Data for Rural Areas of Census 2011

\section{MATERIAL AND METHODS}

11 main variables have been considered for analytical purpose in this study. These 11 main variables are consisting of several sub variables. Very few sub variables are excluded due to lack of importance, insignificant small data values and lesser impact in 2020 corresponding to 2010 .

Dataset obtained by excluding a very few sub variables in the above process and finally it consists of 78 variables.

$x_{i j k}=$ Value of $j^{\text {th }}$ subvariable of $i^{\text {th }}$ main variable for the $k^{\text {th }}$ district. $i=1(1) 11, \quad j=1(1) n(i) ; k=1(1) 18$ We define for fixed $\mathrm{i}$ and $\mathrm{k}$, $p_{i j k}=x_{i j k} / \sum_{j=1}^{n(i)} x_{i j k} ; \mathrm{i}=1(1) 11$ and $\mathrm{k}=1(1) 18 ., \mathrm{n}(\mathrm{i})=$ number of sub variables in ith main variable.

$1^{\text {st }}$ Method:Considering $w_{i j k}=$ weightage of $p_{i j k}=1 / \mathrm{N}$, where $\mathrm{N}=\operatorname{sum}$ of $\mathrm{n}(\mathrm{i})=78$

$$
\text { Index (ik) }[\mathbf{I 1}(\mathbf{i k})]=\frac{\sum P_{i j k}^{W_{i j k}}}{\sum P_{i j k}^{W_{i j k}}+\sum\left(1-P_{i j k}\right)} W_{i j k}
$$

$2^{\text {nd }}$ Method): Considering $w_{i j k}=$ weightage of $p_{i j k}$

Weightage has been considered as shown above in Table 1.1. Here the weightage of variable varies unlike the first method.

$$
\operatorname{Index}(\boldsymbol{i k})[\mathrm{I} 2(\mathrm{ik})]=\frac{\sum \boldsymbol{P}_{i j k}^{W_{i j k}}}{\sum \boldsymbol{P}_{i j k}^{W_{i j k}}+\sum\left(1-\boldsymbol{P}_{i j k}\right)^{W_{i j k}}}
$$

$3^{r d}$ Method: Considering $w_{i j k}=$ weightage of $p_{i j k}=1 / \mathrm{N}$, where $\mathrm{N}=78$

$$
\operatorname{Index}(\mathbf{i k})[\mathbf{I 3}(\mathbf{i k})]=\frac{\prod P_{i j k}^{W_{i j k}}}{\prod_{i j k}^{W_{i j k}}+\Pi\left(1-P_{i j k}\right)^{W_{i j k}}}
$$

$4^{\text {th }}$ Method: Considering $w_{i j k}=$ weightage of $p_{i j k}$

Weightage has been considered as shown above in Table 1.1. Here the weightage of variable varies unlike the first method.

$$
\boldsymbol{I n d e x}(\boldsymbol{i k})[\boldsymbol{I 4}(\boldsymbol{i k})]=\frac{\prod P_{i j k}^{W_{i j k}}}{\prod P_{i j k}^{W_{i j k}}+\prod\left(1-P_{i j k}\right)^{W_{i j k}}}
$$

Table - 2.1 showing the ranks (R1) of the districts based on $1^{\text {st }}$ method

\begin{tabular}{|l|l|l|l|}
\hline Rank(R1) & District code & District name & Index Value(I1) \\
\hline 1 & 338 & Hugli & 0.63324 \\
\hline 2 & 341 & Haora & 0.63089 \\
\hline 3 & 335 & Barddhaman & 0.62953 \\
\hline 4 & 344 & PaschimMedinipur & 0.62829 \\
\hline 5 & 339 & Bankura & 0.62820 \\
\hline 6 & 334 & Birbhum & 0.62589 \\
\hline 7 & 336 & Nadia & 0.62568 \\
\hline 8 & 337 & North Twenty Four Parganas & 0.62559 \\
\hline 9 & 327 & Darjiling & 0.62534 \\
\hline 10 & 345 & PurbaMedinipur & 0.62519 \\
\hline 11 & 340 & Puruliya & 0.62512 \\
\hline 12 & 343 & South Twenty Four Parganas & 0.62474 \\
\hline 13 & 328 & Jalpaiguri & 0.62432 \\
\hline 14 & 331 & DakshinDinajpur & 0.62429 \\
\hline 15 & 333 & Murshidabad & 0.62367 \\
\hline 16 & 330 & Uttar Dinajpur & 0.62352 \\
\hline 17 & 332 & Maldah & 0.62275 \\
\hline 18 & 329 & Koch Bihar & 0.62166 \\
\hline
\end{tabular}


Ranking of Districts of West Bengal by an Index Developed Through Probabilistic Approach Based on Household Data for Rural Areas of Census 2011

Table -2.2 showing the ranks(R2) of the districts based on $2^{\text {nd }}$ method

\begin{tabular}{|l|l|l|l|}
\hline Ranking(R2) & District code & District name & Index value(I2) \\
\hline 1 & 338 & Hugli & 0.17622 \\
\hline 2 & 327 & Darjiling & 0.16936 \\
\hline 3 & 341 & Haora & 0.16813 \\
\hline 4 & 335 & Barddhaman & 0.16754 \\
\hline 5 & 337 & North Twenty Four Parganas & 0.15868 \\
\hline 6 & 336 & Nadia & 0.15862 \\
\hline 7 & 345 & PurbaMedinipur & 0.15272 \\
\hline 8 & 339 & Bankura & 0.15219 \\
\hline 9 & 344 & PaschimMedinipur & 0.1506 \\
\hline 10 & 333 & Murshidabad & 0.14947 \\
\hline 11 & 334 & Birbhum & 0.14809 \\
\hline 12 & 343 & South Twenty Four Parganas & 0.14807 \\
\hline 13 & 331 & DakshinDinajpur & 0.14739 \\
\hline 14 & 328 & Jalpaiguri & 0.14724 \\
\hline 15 & 332 & Maldah & 0.14575 \\
\hline 16 & 329 & Koch Bihar & 0.14505 \\
\hline 17 & 330 & Uttar Dinajpur & 0.14409 \\
\hline 18 & 340 & Puruliya & 0.14289 \\
\hline
\end{tabular}

Table - 2.3 showing the ranks (R3) of the districts based on $3^{\text {rd }}$ method

\begin{tabular}{|l|l|l|l|}
\hline Rank(R3) & District code & District name & Index value(I3) \\
\hline 1 & 337 & North Twenty Four Parganas & 0.04513 \\
\hline 2 & 338 & Hugli & 0.04453 \\
\hline 3 & 335 & Barddhaman & 0.04254 \\
\hline 4 & 327 & Darjiling & 0.04249 \\
\hline 5 & 336 & Nadia & 0.04104 \\
\hline 6 & 341 & Haora & 0.04092 \\
\hline 7 & 343 & South Twenty Four Parganas & 0.03844 \\
\hline 8 & 345 & PurbaMedinipur & 0.03829 \\
\hline 9 & 333 & Murshidabad & 0.03652 \\
\hline 10 & 332 & Maldah & 0.03405 \\
\hline 11 & 328 & Jalpaiguri & 0.03328 \\
\hline 12 & 344 & PaschimMedinipur & 0.03297 \\
\hline 13 & 334 & Birbhum & 0.03149 \\
\hline 14 & 331 & DakshinDinajpur & 0.03140 \\
\hline 15 & 339 & Bankura & 0.03026 \\
\hline 16 & 330 & Uttar Dinajpur & 0.02933 \\
\hline 17 & 340 & Puruliya & 0.02662 \\
\hline 18 & 329 & Koch Bihar & 0.02653 \\
\hline
\end{tabular}

Table -2.4 showing the ranks $(R 4)$ of the districts based on $4^{\text {th }}$ method

\begin{tabular}{|l|l|l|l|}
\hline Rank(R4) & District code & District name & Index value(I4) \\
\hline 1 & 338 & Hugli & $8.52389 \mathrm{E}-14$ \\
\hline 2 & 327 & Darjiling & $4.97226 \mathrm{E}-14$ \\
\hline
\end{tabular}


Ranking of Districts of West Bengal by an Index Developed Through Probabilistic Approach Based on Household Data for Rural Areas of Census 2011

\begin{tabular}{|l|l|l|l|}
3 & 337 & North Twenty Four Parganas & $3.15052 \mathrm{E}-14$ \\
\hline 4 & 335 & Barddhaman & $2.95542 \mathrm{E}-14$ \\
\hline 5 & 341 & Haora & $2.01082 \mathrm{E}-14$ \\
\hline 6 & 336 & Nadia & $1.13277 \mathrm{E}-14$ \\
\hline 7 & 345 & PurbaMedinipur & $2.51883 \mathrm{E}-15$ \\
\hline 8 & 343 & South Twenty Four Parganas & $2.30413 \mathrm{E}-15$ \\
\hline 9 & 333 & Murshidabad & $1.76082 \mathrm{E}-15$ \\
\hline 10 & 332 & Maldah & $6.70960 \mathrm{E}-16$ \\
\hline 11 & 328 & Jalpaiguri & $5.98242 \mathrm{E}-16$ \\
\hline 12 & 344 & PaschimMedinipur & $5.51777 \mathrm{E}-16$ \\
\hline 13 & 334 & Birbhum & $3.78713 \mathrm{E}-16$ \\
\hline 14 & 339 & Bankura & $3.28377 \mathrm{E}-16$ \\
\hline 15 & 331 & DakshinDinajpur & $2.40617 \mathrm{E}-16$ \\
\hline 16 & 330 & Uttar Dinajpur & $1.53489 \mathrm{E}-16$ \\
\hline 17 & 329 & Koch Bihar & $5.43194 \mathrm{E}-17$ \\
\hline 18 & 340 & Puruliya & $2.88899 \mathrm{E}-17$ \\
\hline
\end{tabular}

Table 2.5 showing final table of ranking

\begin{tabular}{|l|l|l|l|l|l|}
\hline Code & District name & R1 & R2 & R3 & R4 \\
\hline 339 & Bankura & 5 & 8 & 15 & 14 \\
\hline 335 & Barddhaman & 3 & 4 & 3 & 4 \\
\hline 334 & Birbhum & 6 & 11 & 13 & 13 \\
\hline 331 & DakshinDinajpur & 14 & 13 & 14 & 15 \\
\hline 327 & Darjiling & 9 & 2 & 4 & 2 \\
\hline 341 & Haora & 2 & 3 & 6 & 5 \\
\hline 338 & Hugli & 1 & 1 & 2 & 1 \\
\hline 328 & Jalpaiguri & 13 & 14 & 11 & 11 \\
\hline 329 & Koch Bihar & 18 & 16 & 18 & 17 \\
\hline 332 & Maldah & 17 & 15 & 10 & 10 \\
\hline 333 & Murshidabad & 15 & 10 & 9 & 9 \\
\hline 336 & Nadia & 7 & 6 & 5 & 6 \\
\hline 337 & North Twenty Four Parganas & 8 & 5 & 1 & 3 \\
\hline 344 & PaschimMedinipur & 4 & 9 & 12 & 12 \\
\hline 345 & PurbaMedinipur & 10 & 7 & 8 & 7 \\
\hline 340 & Puruliya & 11 & 18 & 17 & 18 \\
\hline 343 & South Twenty Four Parganas & 12 & 12 & 7 & 8 \\
\hline 330 & Uttar Dinajpur & 16 & 17 & 16 & 16 \\
\hline
\end{tabular}

Table 2.6 Correlation between ranks

\begin{tabular}{|l|l|l|l|l|l|}
\hline \multicolumn{6}{|l|}{ Correlations } \\
\hline & & R1 & R2 & R3 & R4 \\
\hline R1 & Pearson Correlation & 1 & $.981^{* *}$ & $.523^{*}$ & $.833^{* *}$ \\
\hline & Sig. (2-tailed) & & 0.000 & 0.026 & 0.000 \\
\hline R2 & Pearson Correlation & $.981^{* *}$ & 1 & $.542^{*}$ & $.882^{* *}$ \\
\hline & Sig. (2-tailed) & 0.000 & & 0.020 & 0.000 \\
\hline R3 & Pearson Correlation & $.523^{*}$ & $.542^{*}$ & 1 & $.779^{* *}$ \\
\hline
\end{tabular}


Ranking of Districts of West Bengal by an Index Developed Through Probabilistic Approach Based on Household Data for Rural Areas of Census 2011

\begin{tabular}{|l|l|l|l|l|l|} 
& Sig. (2-tailed) & 0.026 & 0.020 & & 0.000 \\
\hline R4 & Pearson Correlation & $.833^{* *}$ & $.882^{* *}$ & $.779^{* * *}$ & 1 \\
\hline & Sig. (2-tailed) & 0.000 & 0.000 & 0.000 & \\
\hline
\end{tabular}

\section{REMARKS}

An attempt has been made to use four different approaches for finding ranks based on House listing and Housing Census Data for rural areas. The approaches are simple average, weighted average, simple geometric mean, weighted geometric mean. Correlation coefficient shows that the methods are not significantly different (Table 2.6). From the Table 2.5, it is observed that the optimal district is either Hugli or Koch Bihar. The next better district is Barddhaman, Haora, etc. The deviation of rankings is maximum for the District Bankura, Paschim Medinipur, Malda, Birbhum, etc.

\section{ACKNOWLEDGEMENT}

Authors want to acknowledge - Office of the Registrar General, India and Director of Census Operations, West Bengal.

Disclaimer: The findings of this paper is the view of the Authors. It has nothing to do to any Organization.

\section{REFERENCE}

1) Bhadra P P and Ghara T K (2020): Ranking Of Districts Of West Bengal By An Index Developed Through Household Data For Rural Areas Of Census 2011 (accepted IJRSR)

2) Bhadra P P and Ghara T K (2020): Ranking of Districts of West Bengal by an Index Developed Through Census 2011 Household Data (Urban \& Total), International Journal of Humanities and Social Science Invention (IJHSSI), Vol-9 No-7 Pp31-36

3) Census 2011: Census of India 2011 Provisional Population Totals Urban Agglomerations and Cities : www. censusindia.gov.in/2011-prov-results/paper2/data_files/India2/1. Data Highlight.pdf

4) Das, B and Mistri, A (2013): Household Quality of Living in Indian States: Analysis of 2011, Census: https://doi.org/10.1177/0975425313477759

5) Directorate of Census Operators, West Bengal.

6) Ghara, T K(2016): Classification of the States of India Based on Higher Education Development Indicators, IOSR Journal of Research \& Method in Education, Volume 6, Issue 6 Ver. I, PP 65-70

7) Jian L, Saha B and Deshpande A(2009) : A Unified Approach to Ranking in Probabilistic Databases

8) Leclerc G, Nelson A and Knapp E B(2000): The use of unit-level census data for research on poverty: a multiscale approach; International Center for Tropical Agriculture (CIAT), Colombia 